\title{
Are numbers special? An overview of chronometric, neuroimaging, developmental and comparative studies of magnitude representation
}

\author{
Roi Cohen Kadosh ${ }^{\mathrm{a}, 1, *}$, Jan Lammertyn ${ }^{\mathrm{b}, 1}$, Veronique Izard $^{\mathrm{c}, 1}$ \\ ${ }^{a}$ Institute of Cognitive Neuroscience and Department of Psychology, University College London, London WC1N 3AR, UK \\ ${ }^{\mathrm{b}}$ Department of Experimental Psychology, Ghent University, Ghent, Belgium \\ ${ }^{\mathrm{c}}$ Laboratory for Developmental Studies, Harvard University, Cambridge, MA 02138, USA
}

Received 2 April 2007; received in revised form 2 October 2007; accepted 7 November 2007

\begin{abstract}
There is a current debate whether the human brain possesses a shared representation for various types of magnitude such as numerical quantities, physical size, or loudness. Here, we critically review evidence from chronometric, neuroimaging, developmental and comparative fields, and supplement it with a meta-analysis of the neuroimaging data. Together, based on such an integrative overview, we discuss limitations inherent in each approach, and the possibility whether shared, or distinct magnitude representation, or both representations exist.
\end{abstract}

(C) 2007 Elsevier Ltd. All rights reserved.

Keywords: Animal cognition; Human cognition; Development; Magnitude; Neuroimaging; Numerical processing; Parietal lobe; Representation; Neural specialization

\section{Contents}

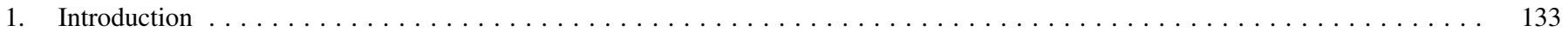

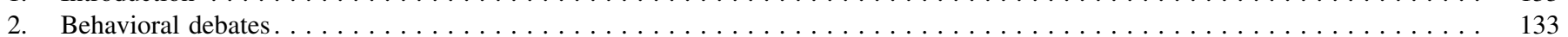

2.1. Similar effect patterns with different kinds of quantity: the distance, size and SNARC effects. . . . . . . . . . 133

2.2. Interaction between symbolic and non-symbolic quantities: the size congruity effect $\ldots \ldots \ldots \ldots$

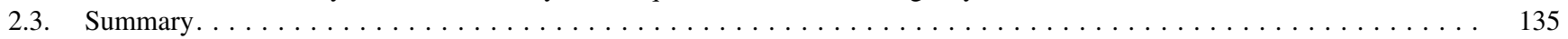

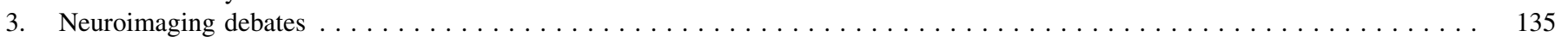

3.1. Evidence for overlap in the neural code for different magnitude dimensions $\ldots \ldots \ldots \ldots \ldots$

3.2. Evidence for separate neural codes for different magnitude dimensions $\ldots \ldots \ldots \ldots \ldots$

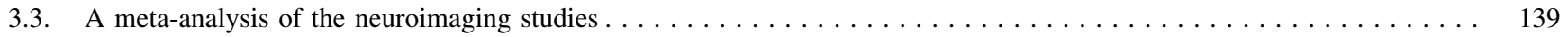

3.4. A comment on methodology: do similar reaction times across tasks reflect identical cognitive resources? . . . . . . 140

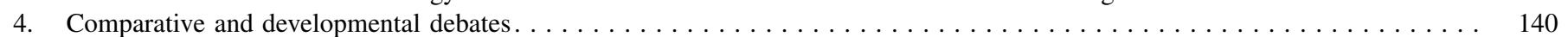

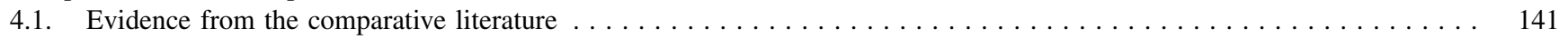

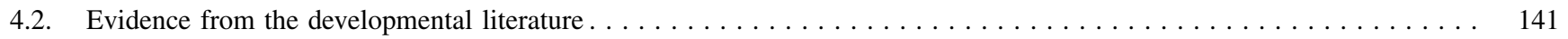

4.2.1. Infants' sensitivity to numerosity versus non-numerical continuous magnitudes $\ldots \ldots \ldots \ldots 1$

4.2.2. Comparison of infants' behavior across different types of magnitudes $\ldots \ldots \ldots \ldots \ldots$

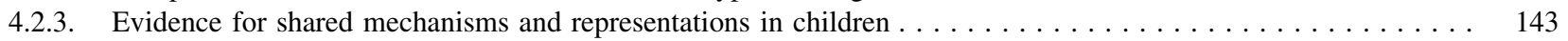

4.2.4. Evidence for shared representation from developmental synaesthesia . . . . . . . . . . . . . . . 143

Abbreviations: ERPs, event-related potentials; fMRI, functional magnetic resonance imaging; IPS, intraparietal sulcus; PET, positron emission tomography; RT, reaction time; SNARCs, spatial numerical association of response code; TMS, transcranial magnetic stimulation.

* Corresponding author. Tel.: +44 20 76701160; fax: +44 2079168517.

E-mail address: r.cohenkadosh@ucl.ac.uk (R. Cohen Kadosh).

${ }^{1}$ The authors have contributed equally to this manuscript. 


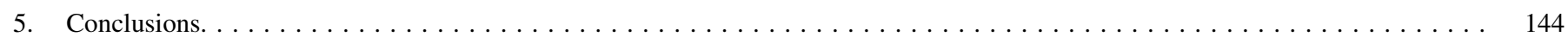

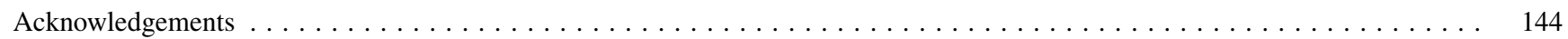

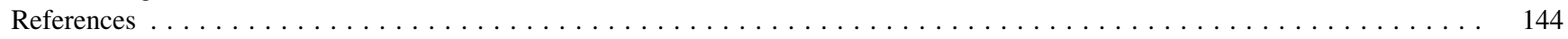

\section{Introduction}

"Number is the within of all things" Pythagoras of Samos

We are incessantly comparing things in our environment. Did we receive the correct amount of change in the cafeteria? Which one of two quarreling brothers is taller? Is the music of the next-door neighbor quieter after we ask him to reduce it? There are countless examples. How do we represent all these different stimuli; how does the human brain process these quantities; and is there only one shared representation for magnitude in the brain, or are different dimensions represented by different neuronal populations in the same brain area? These questions are fundamental to understanding how numerical and other quantity-related information is processed by the brain.

Human processing of numbers has intrigued researchers from various disciplines such as anthropology (Urton and Brezine, 2005), linguistics (Gordon, 2004; Pica et al., 2004; Wiese, 2003), psychology (Gallistel and Gelman, 1992; Gelman and Butterworth, 2005; Moyer and Landauer, 1967), and cognitive neuroscience (Brannon, 2006; Dehaene et al., 2003; Fias et al., 2003; Nieder, 2005). Previous reviews focused on the question whether the same representation exists for different notations of numbers (i.e., the issue of abstract numerical representation; Brannon, 2006; Dehaene et al., 1998). In this paper we focus on the question whether the same representation exists for numbers and other types of magnitude. Namely, we review behavioral, neuroimaging, developmental and comparative studies. By doing so, we try to resolve the question whether we represent numbers in a distinct way (henceforth, distinct magnitude representation), or whether the same representations are involved in the processing of numbers and any other kind of non-numerical magnitude (henceforth, shared magnitude representation). Knowledge about the specificity of numerical processing is of importance in regard to our understanding of the human brain, evolution and the development of numerical understanding. Because our goal is to bring together evidence emanating from different fields of research, we will be considering both the mental processes and representations involved, as well as the neuronal substrates subserving these given processes and representations.

\section{Behavioral debates}

The question whether we have different magnitude representations for each type of quantity information, or alternatively, we possess one unified magnitude system serving all sorts of quantity input formats, has been the focus of much research during the last decade. This research culminated in the ATOM (a theory of magnitude) model introduced by Walsh (2003), which proposes a system of generalized magnitude representations serving diverse quantifiable dimensions (i.e., number, space and time). To investigate the feasibility of such a shared magnitude representation as opposed to a distinct magnitude representation point of view, we will consider in this section behavioral studies that show commonalities between numerical and non-numerical magnitude dimensions. First, we will look at behavioral studies that show similar effect patterns but with different magnitude dimensions. Second, we will consider studies in which interactions between numerical and non-numerical magnitude dimensions are found.

As we will show, evidence emerging from these studies can be interpreted in two ways: either numbers and other nonnumerical magnitudes are represented via a shared magnitude system, or alternatively, the mechanisms used by these dimensions are shared across dimensions in the sense that the same mechanisms (e.g., comparison mechanism, response selection) operate indistinctly on different kinds of magnitude representations.

\subsection{Similar effect patterns with different kinds of quantity: the distance, size and SNARC effects}

In the late 1960s and early 1970s, the idea emerged that mental number processing involves a mental number line on which numerical magnitude is represented (Moyer and Landauer, 1967; Restle, 1970). The spatial organization of numerical information on the number line causes some specific effects. One of the most robust examples in this regard is the numerical distance effect (Moyer and Landauer, 1967): it is easier to compare numbers that are quantitatively farther away from each other than numbers that are quantitatively closer to each other (e.g., people respond faster to the largest number when viewing 8 and 2, compared to when they are presented with the numbers 4 and 2). Another typical effect found when comparing numerical magnitude is the size effect, that is, comparing numbers becomes increasingly difficult the larger they are, even when the distance between them is kept constant (e.g., comparing 8 and 9 is more difficult than comparing 2 and 3). A third effect, which is also based on the close relation between numbers and space, is the SNARC effect (spatial numerical association of response codes) (Dehaene et al., 1993; for reviews see Fias and Fischer, 2004; Gevers and Lammertyn, 2005). It refers to the observation that participants are faster to respond to small numbers with left-hand responses compared to right-hand responses, and faster to respond to large numbers with their right hand than with their left hand. According to Dehaene et al. (1993), this interaction between numbers and space is directly related to the left-to-right orientation of the metaphorical mental number line (smaller numbers on the left, larger numbers on the right), and they suggested that this specific orientation is formed by cultural factors such as general writing direction (see also Zebian, 2005). 
Table 1

Studies investigating or reporting (a) a distance or (b) a size effect on different dimensions and with different stimuli

\begin{tabular}{|c|c|c|c|}
\hline Dimension & Stimuli & Task & Authors \\
\hline \multicolumn{4}{|c|}{ (a) Distance effect } \\
\hline \multirow[t]{4}{*}{ Magnitude } & Digits & Comparison & $\begin{array}{l}\text { Buckley and Gillman (1974), Moyer and } \\
\text { Landauer (1967) and Pinel et al. (2004) }\end{array}$ \\
\hline & Numerosities & Sorting & Crossman (1955) \\
\hline & Numerosities & Comparison & Buckley and Gillman (1974) \\
\hline & Two-digit numbers & Comparison & Restle (1970) \\
\hline \multirow[t]{2}{*}{ Area } & Geometrical shapes & Order comparison & Fulbright et al. (2003) \\
\hline & Size of symbols & Comparison & $\begin{array}{l}\text { Pinel et al. (2004), Cohen Kadosh et al. (2005), } \\
\text { Kaufmann et al. (2005) and Tang et al. (2006) }\end{array}$ \\
\hline Length & Lines & Comparison & $\begin{array}{l}\text { Henmon (1906), Johnson (1939) and Birren and } \\
\text { Botwinick (1955) }\end{array}$ \\
\hline Luminance & Digits with different luminance levels & Comparison & $\begin{array}{l}\text { Cohen Kadosh et al. (2005), Cohen Kadosh } \\
\text { and Henik (2006) and Pinel et al. (2004) }\end{array}$ \\
\hline Social status & Navy ranks/academic occupational positions & Comparison & Chiao et al. (2004) \\
\hline Time & Duration of sequences & Comparison & Dormal et al. (2006) \\
\hline Pitch & Tones of different frequency & Comparison & Henmon (1906) and Rusconi et al. (2006) \\
\hline \multicolumn{4}{|l|}{ (b) Size effect } \\
\hline \multirow[t]{3}{*}{ Magnitude } & Two-digit numbers & Comparison & Restle (1970) \\
\hline & Digits & Comparison & Buckley and Gillman (1974) and Parkman (1971) \\
\hline & Numerosities & Comparison & Buckley and Gillman (1974) \\
\hline Area & Angle width & Comparison & Fias et al. (2003) \\
\hline Length & Line length & Comparison & Fias et al. (2003) \\
\hline
\end{tabular}

All the numerical effects described before are a result of the representation that underlies them. Therefore it is interesting to explore if other non-numerical magnitude dimensions also show the typical effect patterns exposed by numbers. It is clear that even though the distance and size effects are typical examples of behavioral number effects, they are not exclusively observed with numbers (see Table 1). Already in 1906, Henmon exposed pairs of lines to subjects, asking them to decide which is the largest by pressing one of two morse keys. He found that reaction times tend to rise when the discrimination becomes finer (see Welford, 1960). Since then, the distance effect has been shown many times using pairs of lines (e.g., Johnson, 1939; Fias et al., 2003). However, there are numerous examples of other stimuli that show a distance and/or size effect (Table 1). In fact, the distance effect is observable with any psychophysical quantity dimension such as size of geometrical shapes (Fulbright et al., 2003), size of symbols (Cohen Kadosh et al., 2005; Kaufmann et al., 2005; Pinel et al., 2004; Tang et al., 2006), luminance
(Cohen Kadosh and Henik, 2006; Cohen Kadosh et al., 2005; Pinel et al., 2004) and pitch height (Rusconi et al., 2006). In this last study, subjects compared the pitch of variable frequency tones with that of a fixed reference. The result was that subjects were faster and more accurate when the difference between the pitch heights of both tones became larger.

The size effect has also been observed with other stimuli than numbers. For example, Fias et al. (2003) obtained a size effect when subjects compared pairs of angles or pairs of lines. When grouping reaction times (RTs) on the basis of the size of the smallest angle (or line) in the pair, participants responded significantly slower with increasing size (or length).

The same is true for the SNARC effect. Although initially the SNARC effect was found exclusively with numbers as stimuli, later studies showed that non-numerical magnitude information is also spatially coded (see Table 2). For instance, Rusconi et al. (2006) found evidence that the height of tonal information (pitch) is also spatially represented, resulting in the

Table 2

Studies investigating the SNARC effect with different kinds of stimuli

\begin{tabular}{lll}
\hline Stimuli & Task & Authors \\
\hline Numbers & Parity & Dehaene et al. (1993) and Fias et al. (1996) \\
& Phoneme monitoring & Fias et al. (1996) \\
& Orientation detection & Fias et al. (2001) and Lammertyn et al. (2002) \\
& Parity & Fias (2001)
\end{tabular}


so-called SMARC effect (Spatial-Musical Association of Response Codes).

\subsection{Interaction between symbolic and non-symbolic quantities: the size congruity effect}

Showing that homologous effects occur both with numerical and non-numerical quantities does not necessarily imply that both types of magnitude use shared representations. A more convincing finding would be that numerical and non-numerical quantities interact with each other. This is exactly what is found with the size congruity paradigm (Algom et al., 1996; Cohen Kadosh and Henik, 2006; Cohen Kadosh et al., 2007e; Fias et al., 2002; Henik and Tzelgov, 1982; Hurewitz et al., 2006; Pansky and Algom, 1999; Schwarz and Ischebeck, 2003; Tzelgov et al., 1992). In this Stroop-like task, numerical and physical dimensions are varied independently. Suppose that two digits are presented and participants are asked to decide which digit is physically larger while ignoring the numerical values. Commonly, participants cannot ignore the numerical values, which interfere with their physical judgments. That is, participants usually respond slower to incongruent pairs (e.g., 2 4) than to congruent pairs (e.g., 24 ). The same effect is also observed when participants are asked to carry out the opposite task (i.e., compare the numerical value while ignoring the physical size). Furthermore, Cohen Kadosh and Henik (2006) showed that the interaction between symbolic and nonsymbolic quantities is also present between luminance and numbers (although it can be concluded from a recent study (Cohen Kadosh et al., in press-b) that the degree of contrast rather than luminance was manipulated). Together, the multiple findings of the size congruity effect with and between different dimensions suggest that different types of magnitude tap the same magnitude mechanism. Moreover, the distance and the size effects are believed to reflect a process that is derived from the mental number line (Moyer and Landauer, 1967). Hence, additional support for the suggestion that different dimensions interfere with each other as a result of a shared magnitude processing is given by the interaction of the size congruity effect with the distance effect (Cohen Kadosh and Henik, 2006; Cohen Kadosh et al., 2007e; Girelli et al., 2000; Henik and Tzelgov, 1982; Schwarz and Heinze, 1998; Schwarz and Ischebeck, 2003; Tzelgov et al., 1992), and the size effect (Pinhas et al., submitted for publication).

\subsection{Summary}

Together, the observation of similar effects suggests that the semantic representation of numbers is shared with other nonnumerical magnitude dimensions. However, the question is whether this is sufficient evidence. An alternative explanation could be that different quantity dimensions (numerical and nonnumerical) do not share an internal representation, but share mechanisms operating on these representations (e.g., the comparison mechanism). From this point of view, effects like the distance effect or the size effect do not have to emerge at the level of the representation. Namely, they might originate at the level of transition from representation towards the decision and/ or response selection stage where comparison takes place (for the distance effect see Cohen Kadosh et al., in press-a; for the size effect see Verguts et al., 2005; but see Cohen Kadosh et al., in press-c, for challenging this view by supporting the idea that the size effect originates at the level of the representation).

Therefore, the question whether numbers are special is difficult to answer on the basis of behavioral data alone. A complementary way to investigate the assumption of a distinct representation versus the supposition of a shared representation is by looking at the neural substrate underlying the processing of symbolic and non-symbolic quantities.

\section{Neuroimaging debates}

While RT data certainly are informative in the shared versus distinct representation discussion, they might be insufficient to make a real distinction. This is so because stimuli yielding similar response functions can still, theoretically, be processed by distinct mechanisms (Rumelhart and McClelland, 1986). As we will show here, the usage of imaging techniques is of fundamental importance in this respect and can provide information as well as distinguish between these psychological theories.

It is well documented that the intraparietal sulcus (IPS) is involved in processing numerical magnitude (for reviews see Brannon, 2006; Dehaene et al., 2003). It might well be that the IPS is used for coding magnitude in general (Walsh, 2003), whether numerical or non-numerical. Alternatively, the neural populations implementing different magnitudes might be separated (although they can overlap in an imaged voxel, e.g., Cohen Kadosh et al., 2007b). That is, neurons coded for numbers might respond exclusively to numbers.

\subsection{Evidence for overlap in the neural code for different magnitude dimensions}

Several studies argue that the same neural code is shared between different magnitude dimensions because the IPS is equally active when comparing different kinds of magnitudes, not only numbers. For instance, in a positron emission tomography (PET) study, Fias et al. (2003) compared the IPS response to symbolic magnitude representations such as numbers with non-symbolic magnitude representations such as line lengths and angles. In addition, in a functional magnetic resonance imaging (fMRI) study, Cohen Kadosh et al. (2005) compared IPS activation while participants compared two digits on their numerical values, their height, and their luminance. Both studies found that the posterior part of the left IPS was activated by all the comparison tasks. Moreover, in the latter study, the same cluster was also modulated by numerical distance effect, size distance effect, and luminance distance effect. Hence, these studies, by using different stimuli and imaging methods, provided converging evidence for the existence of a shared magnitude neural code.

Although these studies support the idea of a shared magnitude code related to the IPS, they also challenge it by finding a greater activation for numbers in the anterior part of 
the left IPS. However, this finding does not necessarily imply that the anterior part of the left IPS hosts a specific representation of numbers. It could be that such higher activation was due to more cognitive resources for number processing in the anterior IPS (see Section 3.3). For example, Zorzi and Butterworth (1999) suggested that the discrete numerical representation calls for higher processing requirements compared with the analogue representation of physical size. Hence, this activation would not necessarily be magnitude related but stimulus related.

Similar IPS activation patterns, like the one that is observed with numerical tasks, have also been related to response selection demands (Bunge et al., 2002; Cohen Kadosh et al., 2007d,g; Corbetta and Shulman, 2002; Jiang and Kanwisher, 2003). Such a possible explanation was studied directly by Göbel et al. (2004). In their study, participants compared different numbers to a standard (e.g., the digit 5). The control tasks - detecting the presence of a vertical line in numerical or non-numerical stimuli (i.e., line-detection tasks) - were matched for response selection (as indicated by RT) to the numerical comparison task. The results indicated that there were no IPS regions specific for numerical comparison when contrasted with the line-detection task. Such a result seems to question the implication of the IPS in numerical processing, as well as in any task involving a magnitude judgment. However, the control tasks used in this study involved some orientation components, which might have activated the IPS (Fias et al., 2002, 2003). This is in line with the suggestion that the IPS is involved in visuospatial abilities (Cohen Kadosh et al., 2007d; Fischer et al., 2003; Hubbard et al., 2005; Vuilleumier et al., 2004; Zorzi et al., 2002), which are also implicated in number processing (see the SNARC effect in Section 2.1). Therefore, it is not clear whether the lack of number-specific activation was due to controlling for response selection per se. An alternative suggestion is that the lack of number-specific activation was due to the involvement of visuospatial processing, which is part of the numerical characteristics, in the control task.
Similar to Göbel et al.'s (2004) study, Shuman and Kanwisher (2004) conducted a series of experiments in order to test the specificity of the IPS for numbers. However, they failed to find a unique activation for non-symbolic numbers (dots array, i.e., numerosity) when contrasted with nonmagnitude tasks. In Experiment 1, numerical comparison did not show a higher activation in the IPS as compared to color comparison. In Experiment 2, numerosity did not cause fMR adaptation (a reduction in the fMR signal due to the adapted neuronal population when the same stimulus was presented repetitively (Grill-Spector et al., 2006; Grill-Spector and Malach, 2001)) in the IPS. In Experiment 3, comparison of the numerosity of a flash sequence and a dot array versus color comparison did not yield higher activation in the IPS. These results challenge the hypothesis that numerosity is encoded in the IPS by a distinct magnitude representation. Moreover, it is assumed that numerical representation is notation and modality independent (i.e., abstract representation; Brannon, 2006; Dehaene, 1992; Dehaene et al., 1998; McCloskey, 1992). Therefore, this result challenges the idea of a distinct magnitude representation for numerosity in particular, and numbers in general (e.g., digits, number words, numerosity) (but see Piazza et al., 2007). However, it is important to note that in other studies, numerosity adaptation (Cantlon et al., 2006; Piazza et al., 2004), and distance effects (Ansari et al., 2006a) have been observed using similar adaptation paradigms, questioning the absence of any effect in Experiment 2. In addition, the null result in Experiments 1 and 3 can be explained by the comparison of colors according to their luminance level, which might activate the same area as for numbers (Cohen Kadosh et al., 2005) (Fig. 1) (see Castelli et al., 2006; Nieder, 2004, for additional explanations).

To sum up, these studies support the idea that the brain uses the same structures to process all types of magnitudes, independently of the input format. That is, coding in the IPS seems to be for domain-general magnitudes. Alternatively, it is also possible that the magnitudes themselves are represented in

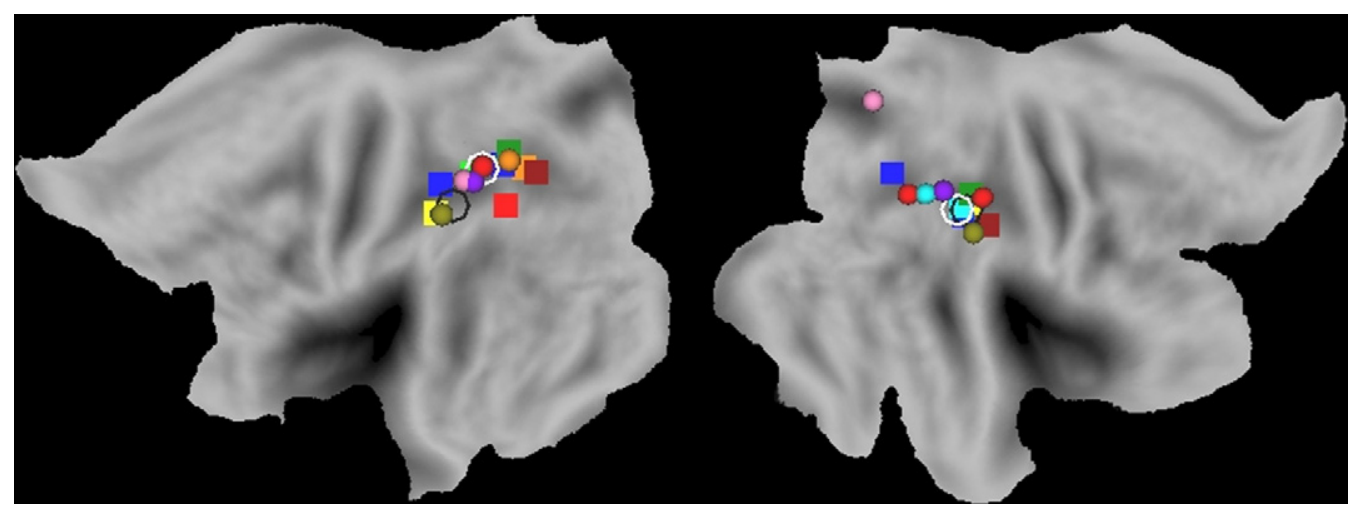

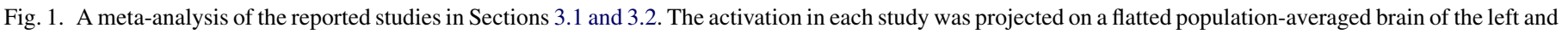

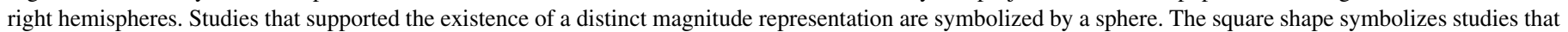

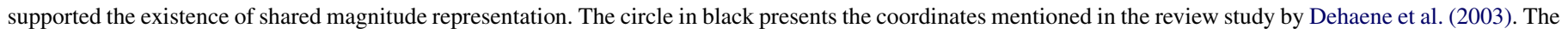

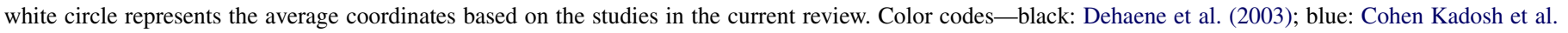

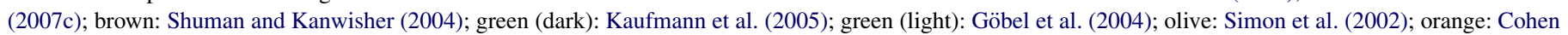

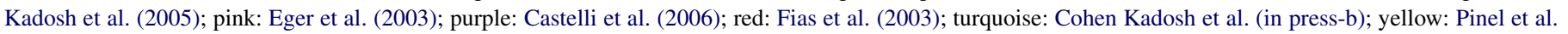
(2004). 
different areas of the brain, depending on the dimension considered, but the IPS hosts a common mechanism involved in processing the comparison between two magnitudes. As all kinds of magnitudes have analogous properties, and presumably their neural codes have analogous properties as well, it is highly possible that the mechanisms operating on these magnitudes are shared across dimensions even if the magnitudes themselves are implemented by distinct neural populations.

As previously mentioned in the behavioral section of this review, a different task that might be used to examine the uniqueness of numbers is the size congruity task (Cohen Kadosh and Henik, 2006; Cohen Kadosh et al., 2007e; Henik and Tzelgov, 1982; Pansky and Algom, 1999; Schwarz and Ischebeck, 2003; Tzelgov et al., 1992). The slowing down of responses (i.e., the size congruity effect) by the irrelevant dimension suggests that processing of numerical and physical dimensions overlap up to a certain point, when selection of the relevant dimension occurs. By pinpointing the stage and the brain area at which conflicts occur, it is possible to differentiate brain areas that are highly specialized from those that are common to several processing streams.

Previous results showed that the interaction between physical and numerical magnitude occurs in the IPS. Namely, fMRI studies revealed that the IPS is modulated both by the distance effect and by the size congruity effect (Kaufmann et al., 2005; Pinel et al., 2004; Tang et al., 2006; although see Ansari et al., 2006b, for different results with number words). For example, Pinel et al. (2004) were the first to use the size congruity paradigm with fMRI, by scanning subjects while they compared size, number, and luminance, which varied orthogonally. They found that the IPS was modulated by both size, and numerical distance effects, with close but not identical peaks of activation. Moreover, the difference between number and size in the IPS was not significant. However, this lack of difference might be due to the fact that manipulation of numerical and physical size occurred continuously and simultaneously. Importantly, size and numbers caused a mutual interference (size congruity effect) at the behavioral and functional levels. Another study found that the interference between numbers and other magnitudes in the IPS is not confined to magnitude with spatial characteristics (i.e., physical size), but also to magnitudes without spatial characteristics (Cohen Kadosh et al., in press-b).

In addition, in an event-related potentials (ERPs) study (Schwarz and Heinze, 1998) it has been shown that the interaction between the physical and numerical dimensions in the size congruity task occurs in pre-response stages. Specifically, the interaction between the physical and numerical magnitudes, as evidenced by the size congruity effect, was reflected by the P300, which indicates a stimulus evaluation and categorization (Kok, 2001; Linden, 2005). In addition, transcranial magnetic stimulation (TMS) to the right IPS of healthy adults reduced the size congruity effect, independent of whether subjects compared the numerical value (and ignored the physical size), or compared the physical size (and ignored the numerical value) (Cohen Kadosh et al., 2007d). Previous studies suggested that difficulties with numerical processing in participants with developmental dyscalculia can be attributed to the right parietal lobe (Geary, 1993; Molko et al., 2003; Rourke, 1993). Importantly, the same results were also obtained when participants with developmental dyscalculia performed the same task (albeit without TMS) (Cohen Kadosh et al., 2007d). Therefore, the automatic processing of numerical value or physical size seems to be equally impaired due to structural abnormality, or stimulation, of the IPS. Together, these results indicate that the IPS hosts a representation of magnitude that is shared by numbers and other dimensions.

These disparate lines of research converge on the conclusion that the human brain is equipped with a shared mechanism to compare numbers and other magnitudes. Moreover, the interference reported between the processing of numbers and other types of magnitudes in the IPS supports the idea that the neural population encoding these magnitudes overlaps, at least partly, in this brain area.

\subsection{Evidence for separate neural codes for different magnitude dimensions}

The shared activation of the IPS by different magnitude dimensions in addition to numbers might suggest a shared representation for magnitude. However, "Absence of evidence is not evidence of absence" (Altman and Bland, 1995; Wilkinson and Halligan, 2004). It might be that a representation for each type of quantity exists, but that most of the different experiments that we reviewed so far were not sensitive enough to capture these dissimilarities.

Simon et al. (2002) found a mosaic of distinct specialized areas in the IPS, including a region for the manipulation of numerical quantities. Functional magnetic resonance images were collected while subjects performed six different tasks: grasping, pointing, saccades, attention, calculation, and phoneme detection. The only area that showed a preference for the calculation task was the anterior part of the left IPS, similar to other later findings (Cohen Kadosh et al., 2005; Fias et al., 2003). One could argue that such activation might be the result of digital representation, which is used for counting (Butterworth, 1999; Di Luca et al., 2006; Göbel et al., 2004; Kansaku et al., 2006), and not from a numerical representation per se. However, the results from control tasks of grasping and pointing, which used digital movement, refute such an argument. Moreover, no area in the parietal lobe showed a joint activation for calculation and grasping, thus, challenging the idea of digital representation as being part of numerical processing (Butterworth, 1999; Di Luca et al., 2006). However, it should be noted that the calculation task yielded the slowest RT among all tasks. This in turn could present a confound between the numerical processing (calculation in this case) and response selection demand (Göbel et al., 2004).

In the absence of a magnitude judgment task, numbers have been found to activate the IPS, in contrast to control stimuli of letters and color patches (Eger et al., 2003). As in numbers, an ordinal component is inherent in letters (A comes before B), and thus letters constitute a particularly well-matched control 
stimulus for numbers. Eger and colleagues tested participants while measuring the percent signal change of blood oxygenation under target detection of numbers, letters or colors, with visual and auditory presentation. For example, participants had to indicate the presence of a target letter while ignoring other non-target letters. The analysis included only the non-target stimuli in order to avoid any confound with the oddball effect that yielded activation in the parietal lobes (Bledowski et al., 2004; Linden, 2005). By using this method, a difference of activation in the brain could not be attributed to components such as response selection. The results indicated greater activation in both the left and right IPS for numbers compared to letters or colors. However, a possible explanation for this exclusive numerical activation can be found in the automaticity of number processing. Namely, the magnitude of numbers is automatically processed even when not relevant to the task, as indicated, for example, by the size congruity effect (Cohen Kadosh and Henik, 2006; Cohen Kadosh et al., 2007e; Henik and Tzelgov, 1982; Pansky and Algom, 1999; Schwarz and Ischebeck, 2003; Tzelgov et al., 1992). In contrast, the degree of automaticity for letters is much smaller than for numbers (Gevers et al., 2003). Hence, such additional activation for numbers can be explained by the fact that domain-general representations of magnitude were automatically accessed in response to numerical stimuli, but not other stimuli.

In a recent fMRI study, Castelli and colleagues examined directly whether there is a specialized mechanism for processing "how many" as compared to "how much" by using non-symbolic stimuli (Castelli et al., 2006). They displayed matched pairs of discrete (i.e., countable) and analogue (i.e., non-countable) stimuli that varied in time or space, while participants had to compare the stimuli according to their color (Which presentation includes more blue or more green?). Although all the stimuli could be processed according to their magnitude, it was found that countable stimuli resulted in stronger IPS activation compared to non-countable stimuli. This result suggests the existence of distinct magnitude codes in the IPS. Nonetheless, the distance effect, which was manipulated in this study, did not show modulation in the countable distinct magnitude clusters. In order to prove that an area is specialized in magnitude processing, it should show not only task-specificity but also effect-specificity, for example, a modulation of the distance effect (Cohen Kadosh et al., 2005). Such a conjunction of task-specificity and distance effect would add additional support to the conclusion that this area is involved in magnitude processing, as reflected by the distance effect, and not another cognitive operation. Hence, although the study by Castelli and colleagues was well controlled for various artifacts, it does not allow us to draw strong inference as to the existence of distinct magnitude codes in the IPS. Namely, the differences between the tasks, while not showing any distance effect, might not necessarily have reflected differences in the magnitude representation per se.

An alternative approach is to look at a later stage than the magnitude representation stage and see whether numbers and other dimensions activate different response streams, thus indicating that they were processed separately in the previous stages. In a combined fMRI and ERP study (Cohen Kadosh et al., 2007c) it has been shown that the interaction between size and numerical magnitude, as indicated by the size congruity effect, modulated activity in the IPS, similar to previous studies (Kaufmann et al., 2005; Pinel et al., 2004). However, numerical and size magnitudes were found to interact in the primary motor cortex also. Namely, a region of interest (ROI) analysis of motor cortex activity revealed aberrant activation of the ipsilateral (irrelevant hand) motor cortex in the incongruent condition. Thus, the neural signature of interference was traced up to the motor cortex. However, in the ERP experiment, the effect of cognitive load was examined, in order to probe the degree to which information processing was shared across cognitive systems; with cognitive load operationalized as the numerical distance between the compared numbers (large numerical distance, low cognitive load; small numerical distance, high cognitive load). The fMRI results were supported by a clear effect in the electrodes above the motor cortex (the so-called, lateralized readiness potential (Gratton et al., 1988)). However, such late interaction between numerical value and physical size was found only in the low cognitive load condition. In contrast, in the high load condition, physical and numerical dimensions interacted only at the comparison stage, as indicated by the P300, as in previous studies (Schwarz and Heinze, 1998).

These results seem to indicate that when the processing of both the relevant and irrelevant dimension is relatively easy (i.e., low cognitive load condition), both numerical value and physical size are processed in parallel until response-related stages. While this observation does not necessarily mean that the conflict is resolved at a later response-related stage, it does indicate that both dimensions produce their magnitude in parallel. This finding supports the hypothesis that the human brain has distinct magnitude representations. However, the results also point to the existence of a shared magnitude representation; when the processing of the relevant and irrelevant dimensions is relatively difficult (i.e., high cognitive load condition), the conflict between numerical value and physical size seems to be resolved already at the comparison stage. This might be because the capacity of the strictly number-specific parts of the parietal cortex is exceeded, requiring the recruitment of areas that are dedicated for other magnitudes, such as physical size. Together, these results suggest that the processing of magnitude is supported by both shared and distinct neural substrates. However, the usage of each mechanism depends on task requirements. This idea fits with previous evidence that humans generate numerical representations according to task requirements (Fischer and Rottmann, 2005; Shaki and Petrusic, 2005), and that more than one type of representation can co-exist (although not necessarily at the same time (Cohen Kadosh et al., 2007a)). Note also that the idea of shared and distinct representations for size and numbers is in line with Pinel et al.'s (2004) proposal, who found close but not identical activation peaks for size and numbers in the IPS (although no significant differences were observed between size and numbers in the IPS). However, looking at a later stage of processing (response selection) as in 
Cohen Kadosh et al.'s study (2007c) strengthens the idea that the close activation peaks in Pinel et al.'s study (2004) might have been due to separate representations rather than more overspread activation for general magnitude that included numerical representation. In other words, theoretically, different but overlapping activation peaks could be derived from one representation being a subgroup of another representation, rather than independent representations.

\subsection{A meta-analysis of the neuroimaging studies}

In order to further explore the question of specialization in the parietal lobes, we projected the activation from all the above-mentioned imaging studies onto a population-averaged human brain by using Caret (http://brainmap.wustl.edu/caret, Van Essen et al., 2001), and SumDB (http://sumsdb.wustl.edu:8081/sums/directory.do?id=636032, Van Essen, 2002). The spatial distribution of the different studies in the IPS is presented in Fig. 1 (see also Table 3) and gives a strong impression against a systematic division between studies that support the idea of distinct magnitude representation (spheres) and studies that support the idea of shared magnitude representation (squares). We further examined this issue by using inferential statistics. First, we transformed all the studies that used different stereotaxic space to a common stereotaxic space (i.e., according to Talairach and Tournoux, 1988). Next, we entered all activations in the parietal lobes, separately for left and right hemispheres, into a $3 \times 2$ analysis of variance (ANOVA), with Talairach coordinates (TCs; $X, Y, Z$ ) and evidence for numerical specialization (yes, no) as factors. The main effect for specialization, as well as the interaction between TC and specialization, was far from significant (all $F$ values $<1$; all $p$ values $>.4$ ).

In addition, we also examined whether our meta-analysis corresponded to a previous meta-analysis in the field (Dehaene et al., 2003). For the right hemisphere, it seems that both our coordinates and the ones obtained by Dehaene et al. fall in close proximity. This is not the case for the left hemisphere. The emergence of this difference might have multiple sources: The meta-analysis by Dehaene et al. (2003) included different tasks. In total, only four studies in their analysis involved comparison of quantity tasks. In the current analysis, we used a larger amount of studies, which is more homogenic in terms of the tasks used.

The lack of any spatial organization in studies that provided support for or against the idea of distinct magnitude representation might be due to several reasons. First, as shown by part of the neuroimaging studies that we presented above, the neuronal populations for different magnitude dimensions are closely localized, and only sophisticated techniques/ analysis can disentangle the different magnitudes. Second, we believe that the different tasks that were used could lead to

Table 3

Activation foci for the meta-analysis presented in Fig. 1

\begin{tabular}{|c|c|c|c|c|c|c|c|c|}
\hline \multirow[t]{3}{*}{ Reference } & \multirow{3}{*}{$\begin{array}{l}\text { Are numbers } \\
\text { special? }\end{array}$} & \multirow[t]{3}{*}{ Task } & \multicolumn{6}{|c|}{ Coordinates of maxima } \\
\hline & & & \multicolumn{3}{|l|}{ Left } & \multicolumn{3}{|c|}{ Right } \\
\hline & & & $X$ & $Y$ & $Z$ & $X$ & $Y$ & $Z$ \\
\hline Fias et al. (2003) & No & Number and line and angle comparison & -36 & -63 & 57 & & n.s. & \\
\hline Cohen Kadosh et al. (2005) & No & Number and size and luminance distance effect & -24 & -58 & 38 & & n.s. & \\
\hline Göbel et al. (2004) & No & Numerical comparison and response selection & -28 & -44 & 48 & & n.s. & \\
\hline Shuman and Kanwisher (2004) & No & Number processing vs. shape/color processing & -27 & -62 & 34 & 50 & -32 & 51 \\
\hline Pinel et al. (2004) & No & Size congruity effect (numbers \& size) & -48 & -36 & 50 & 51 & -29 & 47 \\
\hline Cohen Kadosh et al. (2007c) & No & Size congruity effect (numbers and size) & $\begin{array}{l}-37 \\
-26\end{array}$ & $\begin{array}{l}-37 \\
44\end{array}$ & $\begin{array}{l}43 \\
-55\end{array}$ & $\begin{array}{l}38 \\
25\end{array}$ & $\begin{array}{l}-44 \\
-65\end{array}$ & $\begin{array}{l}45 \\
38\end{array}$ \\
\hline Kaufmann et al. (2005) & No & Number and size distance effect & -24 & -56 & 51 & 28 & -40 & 46 \\
\hline Cohen Kadosh et al. (in press-c) & No & Size congruity effect (numbers and luminance) & & n.s. & & 35 & -43 & 35 \\
\hline Mean & No & & -31 & -51 & 47 & 38 & -42 & 44 \\
\hline S.D. & No & & 8 & 11 & 7 & 11 & 13 & 6 \\
\hline Simon et al. (2002) & Yes & Calculation & -48 & -40 & 50 & 51 & -40 & 50 \\
\hline Eger et al. (2003) & Yes & Number vs. letter and color target detection & -32 & -45 & 32 & 32 & -55 & 30 \\
\hline Fias et al. (2003) & Yes & Number vs. line and angle comparison & -32 & -50 & 52 & $\begin{array}{l}34 \\
36\end{array}$ & $\begin{array}{l}-60 \\
-35\end{array}$ & $\begin{array}{l}40 \\
44\end{array}$ \\
\hline Cohen Kadosh et al. (2005) & Yes & Number vs. size and luminance comparison & -25 & -56 & 43 & & n.s. & \\
\hline Castelli et al. (2006) & Yes & Discrete processing vs. analogue processing & -24 & -44 & 46 & 32 & -53 & 50 \\
\hline Cohen Kadosh et al. (in press-c) & Yes & Size congruity effect (numbers and luminance) & & n.s. & & 32 & -53 & 39 \\
\hline Mean & Yes & & -32 & -47 & 47 & 36 & -49 & 42 \\
\hline S.D. & Yes & & 9 & 6 & 8 & 7 & 9 & 7 \\
\hline Overall mean & & & -31 & -50 & 45 & 37 & -46 & 42 \\
\hline Overall S.D. & & & 8 & 9 & 7 & 9 & 11 & 6 \\
\hline
\end{tabular}


different foci of activation (Cohen Kadosh et al., 2007a, in press-b; Orban et al., 1996), and therefore obscure spatial distribution in studies that gave support for or against the existence of distinct magnitude representation. Our last point is a methodological problem: different analyses can lead to different foci of activation (e.g., Cohen Kadosh et al., 2007b, in press-b).

To sum up, the results reviewed so far suggest that the IPS hosts overlapping domain-general and domain-specific neural populations in human adults for numbers and different magnitudes. The next section will examine the question of neuronal specialization for numerical processing from developmental and comparative perspectives. However, before we proceed to the next section we would like to give a short comment on methodological issues.

\subsection{A comment on methodology: do similar reaction times across tasks reflect identical cognitive resources?}

It is a commonly held view among cognitive psychologists and neuroscientists that in order to examine the commonalities and differences among dimensions and tasks, RT and accuracy should be equal across tasks. Equalizing the RTs is believed to keep the mental effort the same for different tasks. This is a critical point for imaging studies since some brain areas, especially the IPS, show modulation as a function of increased task difficulty (e.g., Göbel et al., 2004). However, there are at least two ways to match reaction times. One is to match the RT for each subject by conducting an individual pilot study prior to the experiment (Pinel et al., 2004). A second way is to match the overall RT for the group of subjects based on a pilot study of different subjects (Cohen Kadosh et al., 2005). While the first method is superior since it leads to equal RTs at the individual level and therefore at the group level, the second method reduces other factors such as training that can alter or affect the activations of interest (Cohen Kadosh et al., in press-b; Poldrack, 2000).

Another logic problem is the tendency to assume that comparable RTs between tasks reflect identical cognitive resources for each component within the tasks (e.g., the same response selection demands). It could be that one task involves greater cognitive resources at stage $X$ and less at stage $Y$, while another task requires less cognitive resources at stage $X$ and more at stage $Y$. The overall RTs in these cases would be similar, but the degree of neuronal activity at the different stages would be different. For example, in the aforementioned studies of Cohen Kadosh et al. (2005) and Pinel et al. (2004), it could be the case that physical size required more effort (and time) to be recognized while numbers took more effort (and time) to be compared. Such an assumed pattern could, in theory, explain why a stronger activation was found for size comparison in the occipitotemporal areas (Cohen Kadosh et al., 2005; Pinel et al., 2004), and for numbers in the IPS (Cohen Kadosh et al., 2005; Fias et al., 2003; Tang et al., 2006). It is hard to disprove this claim by using fMRI alone, which has a coarse temporal resolution. To find an answer to this problem, using other techniques with an excellent temporal resolution, such as ERP, will be essential.

\section{Comparative and developmental debates}

Human infants and animals represent numerosities in a similar format to that of human adults (Cantlon and Brannon, 2006; Dehaene, 1997). For example, a distance effect for numerosities, similar to the effect exhibited by adults, has also been observed in animals (Brannon and Terrace, 1998; Cantlon and Brannon, 2006), and human infants (Lipton and Spelke, 2003; $\mathrm{Xu}$ and Arriaga, 2007; $\mathrm{Xu}$ and Spelke, 2000). Furthermore, some recent imaging results have revealed a developmental continuity in the neural substrate underlying numerosity processing, with a sensibility to numerosities in the right IPS in 4-year-old children (Cantlon et al., 2006), and activation of the left IPS increasing over development (Ansari and Dhital, 2006; Rivera et al., 2005). In the macaque, number sensitive neurons have been recorded in a possible homologue of this area (Nieder et al., 2006; Nieder and Miller, 2004; for reviews see Brannon, 2006; Dehaene et al., 2004; Nieder, 2005). However, even if infants and animals use the same brain structures to represent numbers, the finer neural structures might not resemble the functional organization of the IPS in human adults. Whereas the results reviewed so far suggest that the IPS hosts overlapping domain-general and domain-specific neural populations in human adults, these neural populations might not develop at the same time in children.

Beside the traditional question whether infants and animals can represent numbers at all, the proposed co-existence of shared and distinct magnitude representations in adults thus raises a new question about the development and evolution of numerical processing; namely, which mechanism develops first? One possibility is that shared magnitude representations are present before specific representations. In this case, various magnitudes are represented from infancy, with an archaic, preliterate, non-symbolic system that processes magnitude in general. Later on, the child would develop neuronal circuits dedicated to numerical information, with a possible role of symbolic numerical representations and language (Carey, 2004). This idea is analogues with the Interactive Specialization view (Cohen Kadosh and Johnson, 2007; Johnson, 2001) on human functional brain development. As was implemented in other fields in neuroscience that examined neuronal specialization (e.g., face perception), it might be that at the beginning of the development there is a lack of neuronal specialization for magnitude representation. Later, following interactions between different cortical and sub-cortical areas there will be increased selectivity (fine tuning) in the activation of neuronal substrates in the IPS for specific magnitudes. However, it might be that following cognitive load the recruitment of other magnitude representations, which follows similar principles of representations, occurs.

Alternatively, it may be that specialized representations develop first in the human brain, and shared magnitude processing mechanisms emerge later, as an economical solution making use of the high similarity between the different 
magnitude representations. As for animals, they may have the same mechanism for either shared or distinct numerical representations as infants and young children, or both systems like human adults, unless language and symbols are a prerequisite for the human brain maturing to the adult state.

\subsection{Evidence from the comparative literature}

The question whether numerical representation is domainspecific in animals was addressed in the 1980s by Meck and Church (1983), who suggested that time and numerosity are processed by the same mechanism. They trained rats to associate two auditory sequences, differing both in number of elements ( 2 vs. 8 ) and in time ( $2 \mathrm{~s}$ vs. $8 \mathrm{~s}$ ), to two different levers. In two different subsequent sessions, they tested how rats would generalize their responses to stimuli of intermediate numerosity or time. In the numerosity condition, all the test stimuli had the same time but varied in numerosity, whereas in the timing condition, the stimuli all had the same numerosity but varied in time. For each trial, rats had to select one of the two levers they were previously trained with, thus indicating whether they represented the current test stimulus as more similar to the smallest (shortest) of the stimuli presented in the training phase or to the largest (longest) one. The response curves obtained in the timing and numerosity conditions were strictly identical, in line with Meck and Church's hypothesis of a shared mechanism for time and numerosity. Perhaps more convincing, in subsequent experiments they investigated the effect of methamphetamine on the rats' judgments of time and numerosity (Church and Meck, 1984). The drug induced a bias in perception, which still resulted in strictly identical response curves between numerosity and time. This last result strongly supports Meck and Church's interpretation that number and time processing rely on the same mechanism.

Gallistel and Gelman (2004) adopted a more radical interpretation of Meck and Church's (1983) and Church and Meck's (1984) results. They proposed that time and numbers are actually represented in the same format, that is, as continuous quantities (real numbers) (Gallistel and Gelman, 2004). Besides Meck and Church's results, Gelman and Gallistel's hypothesis was also inspired by the ability of rats to combine information of number and time, for example, when rats computed a rate from duration and numerosity, or multiplied a rate by a reward magnitude to estimate the amount of expected reward (Leon and Gallistel, 1998).

At the brain level, neurons sensitive to numerosity have been observed in the macaque's brain, in the IPS and also in the prefrontal lobes (Nieder et al., 2002, 2006; Nieder and Miller, 2003, 2004; Roitman et al., 2007). Each of these neurons is tuned to a preferred numerosity value (e.g., 4), but also fires when close numerosities are presented (3 and 5,6), and thus represent numbers only approximately, in a format which would be suitable to encode continuous quantities. Recently, Tuduscius and Nieder (2007) tested whether the numerositysensitive neurons in the IPS were also sensitive to line length (a continuous variable). They found that the populations encoding these two attributes overlapped partially: 11.5 and $10 \%$ of the neurons were activated for physical size and numerosity, respectively; whereas, $3.5 \%$ fired for both physical size and numerosity. These results are in accordance with the presence of partially overlapping populations for numerosity and other dimension in the IPS of human adults. However, a possible drawback in the case of neurophysiological experiments, as well as with most animal studies, is that the monkeys must undergo massive training prior to the experiments, which might cause changes in neuronal tissue during this time (e.g., PascualLeone et al., 2005; Poldrack, 2000; but see Roitman et al., 2007).

In summary, the current behavioral, pharmacological and neurophysiological data seem to support the idea that for animals, numbers are not special in the sense that the same format of representation seems to be used for discrete numerosity as well as for any continuous quantity. More precisely, single-cell neurophysiology data reveals a functional organization close to the situation in human adults: different types of magnitudes are represented by partially overlapping and partially distinct populations of neurons.

\subsection{Evidence from the developmental literature}

\subsubsection{Infants' sensitivity to numerosity versus non- numerical continuous magnitudes}

Until very recently the question whether numerical representations in infants are unique or shared with other magnitude dimensions has not been investigated. Instead, since Piaget's pioneer work on young children's judgment of equality (Piaget, 1952), research has focused on the ability of children and infants to estimate the numerosity of sets, and on whether their numerical representations are abstract or depend on the modality of presentation of the stimuli (Barth et al., 2005).

In this vein, a related question was whether infants would still succeed in extracting numerosity in some extreme conditions when non-numerical magnitude-related parameters, such as total amount of substance in the display, or size of each element, were controlled. In this line of research, infants as young as 6 months old have been found to be sensitive to variations in numerosity when either the total amount of substance, the total contour length (Xu and Spelke, 2000; Xu and Arriaga, 2007) or both (McCrink and Wynn, 2004) are controlled. However, because of the severe constraints inherent in developmental research, and despite extreme precautions taken by the authors, in any study it is always possible to extract a non-numerical parameter that is actually confounded with number (Mix et al., 2002), and this leaves open the possibility that infants do not represent numerosity per se, but instead they might always represent general non-numerical magnitude, through a combination of several magnitude cues (e.g., total area, total contour length).

Some observations support the interpretation that infants might not represent numerical information per se, at least in the small number range. For instance, infants habituated to a given numerosity (either 2 or 3 ) do not react to a change in numerosity when the total continuous extent of the display is held constant (Clearfield and Mix, 1999; Feigenson et al., 2002); rather, they 
react when the total continuous extent changes, even when numerosity stays constant. On the other hand, Brannon et al. (2004) have applied the same paradigm to large numerosities and observed a reaction to numerosity but no reaction to the continuous extent, thus showing that numerosity is more salient than non-numerical quantities for arrays containing large number of items. Also in the small number range, Feigenson (2005) discovered that infants can be driven to attend to numerosity over continuous extent, when the elements of the displayed sets present noticeable individual features, such as fur or antennas on characters. These last results show that infants can encode numerical information for sets of any size, as well as the total continuous extent of the sets, for small sets only. However, in the conditions used in most experiments (arrays of similar objects), numerical information is not salient to them; therefore they do not respond to number.

Finally, infants' performance when discriminating numerosities has been found to converge across modalities and for very different types of displays (simultaneously presented visual arrays vs. auditory sequences). These results support the hypothesis that infants can represent numerosities and not only continuous attributes. In the visual modality, infants discriminate large numerosities, and their performance depends on the ratio between the numbers to be discriminated ( $\mathrm{Xu}$ and Spelke, 2000): at 6 months, they can discriminate numerosities in the visual modality in a 1:2 ratio ( 16 vs. 32,8 vs. 16), but they fail to discriminate $2: 3$ ratios (16 vs. 24,8 vs. 12). Infants' performance is identical in the auditory modality (Lipton and Spelke, 2003). Moreover, the precision with which numerosities are discriminated increases with age, with a similar trend in both modalities (Xu and Arriaga, 2007; Lipton and Spelke, 2003). In addition, in the small number range, infants have been shown to be able to compare stimuli across modalities (visual and auditive modalities: Starkey et al., 1983, 1990; but see Moore et al., 1987; Mix et al., 1997, for failures to replicate; Kobayashi et al., 2005; visual and haptic modalities: Féron et al., 2006). The fact that discrimination performance is identical across modalities might be coincidental or it could reflect the evolution of a domain-general comparison process. However, infants' ability to compare numerosities across modalities clearly shows that they are representing numerosities and not only continuous quantities.

\subsubsection{Comparison of infants' behavior across different types of magnitudes}

Beyond the convergence of performances on numerosities across modalities, three recent studies have adopted the same approach to compare representations of magnitude across dimensions (Brannon et al., 2006; vanMarle and Wynn, 2006; see also Feigenson, 2007). Brannon et al. studied infants' ability to detect changes in duration, whereas vanMarle and Wynn investigated their sensibility to changes in area. Interestingly, in both these cases, the threshold where infants started to react to changes corresponded to the thresholds observed in numerosity experiments (Fig. 2). That is, 6-monthold infants reacted to a change in numerosity, area or time when the difference between the two stimuli in each dimension reached the ratio of $1: 2$, but they failed to detect this change for a ratio of 2:3. Furthermore, just as for numbers, the ratio at which they detected a change in duration evolved with age, with 10-month-old infants able to detect changes with a 2:3 ratio in duration as well as in numbers (Brannon et al., 2007).

These last pieces of evidence suggest that representations of magnitude in infants are shared across dimensions, at least between time, number, and area. However, as for adult and animal studies comparing performance across different modalities, these results alone do not specify at which level these representations converge. One possibility is that all magnitudes are represented in the same format (shared magnitude representation). Another possibility is that different
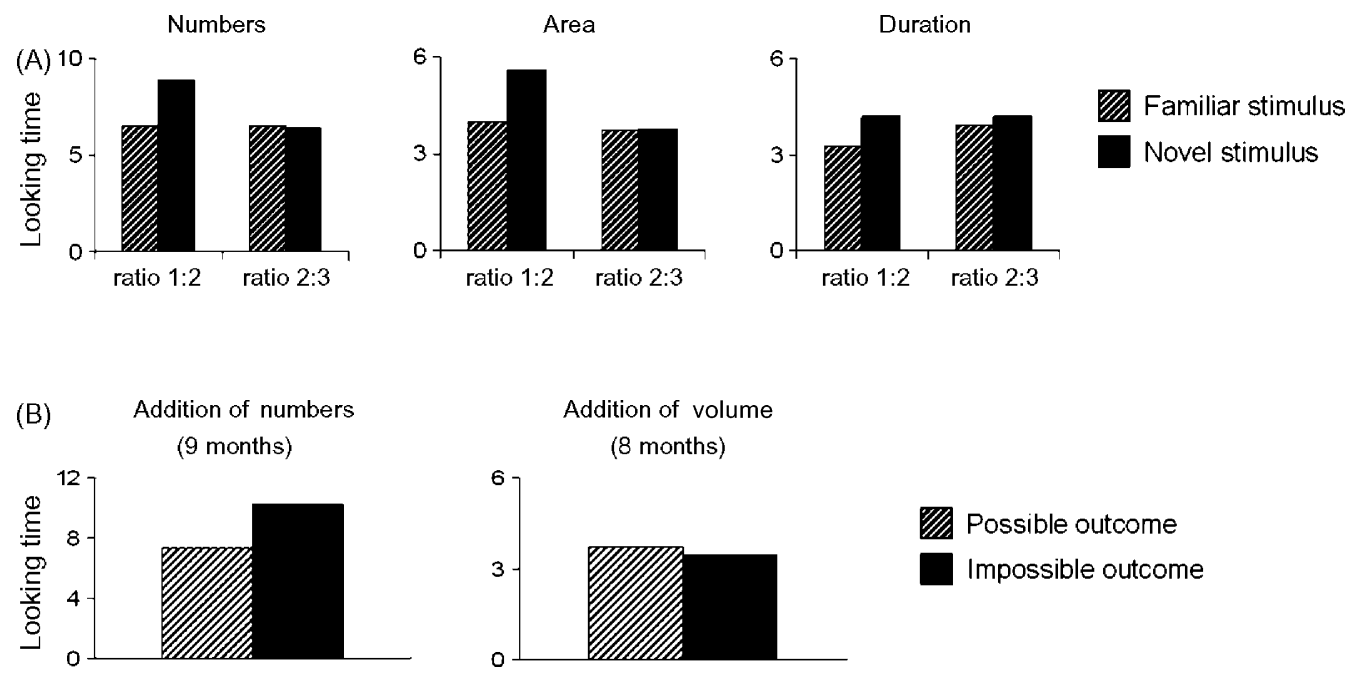

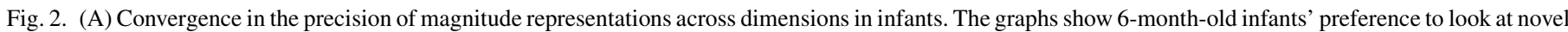

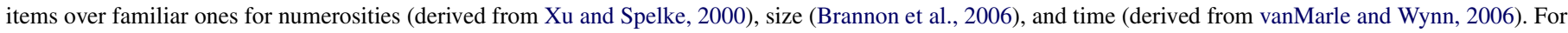

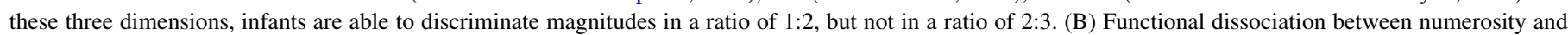

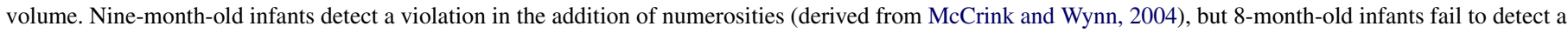
violation for an addition of continuous quantities (Huntley-Fenner et al., 2002). 
types of magnitude are represented in distinct specific formats, but then are compared and processed by a common mechanism (distinct magnitude representations and shared processing mechanisms).

One way to decide whether different dimensions converge to a common format of representations, or whether they are implemented by separate representations but processed by a single comparison mechanism, is to look for possible functional dissociations between dimensions. If all dimensions are implemented in the same format, infants should be able to perform similar operations on all types of magnitudes. In particular, since infants are able to add numerosities, including in the large number range (i.e., up to 10 items) (Wynn, 1992; McCrink and Wynn, 2004), they should be able to add other types of magnitudes with the same precision.

McCrink and Wynn's (2004) demonstration that infants are able to add numerosities in the large number range used the following procedure. At the beginning of each trial, infants were presented with an initial set containing five objects. Then a screen was raised to hide these objects. While the first set was hidden, another set with five additional objects was added behind the screen. At this point, the screen was lowered to uncover either 10 objects (possible outcome) or 5 objects (impossible outcome). In this experiment, objects were constantly changing shape and size to prevent confounds with the number factor. At 9 months of age, infants looked longer at the impossible outcome than at the possible outcome.

On the contrary, Huntley-Fenner et al. (2002) tested 8month-old infants in an analogue situation involving a continuous amount and failed to observe a reaction. In their experiment, infants were first presented with a pile of sand poured onto a stage. Then a screen was raised to hide the initial pile of sand, and additional sand was poured at a different location on the stage. As the screen was lowered, two outcomes could be revealed: either one pile of sand with the same volume as the initial one (impossible outcome), or two piles of sand with the same volume (possible outcome). Infants failed to detect the impossible outcome trials as such.

These two experiments suggest the existence of a dissociation at the functional level between the representations of numerical magnitude and the representations of continuous magnitude. Therefore, convergence across dimensions for the discrimination tasks could be explained by the presence of a shared comparison mechanism, which operates on differentiated magnitude representations. Additional tests are required to confirm the existence of such a dissociation, with more systematically comparable situations across experiments. In the additional experiments cited here, the ratio between the possible and impossible outcomes was 1:2 in the case of the number experiment; and 1:2 in volume in the continuous quantity experiment, which must have resulted in a smaller ratio for apparent area. Moreover, the two sets were merged in the number experiments, while the sand was poured into two separated piles in the continuous outcome experiment. Despite these differences, the two experiments seem to be comparable enough to argue in favour of a dissociation between magnitude representation formats, while the convergence of performance across dimensions shows the existence of a common comparison mechanism operating on these dissociated magnitude representations.

\subsubsection{Evidence for shared mechanisms and representations in children}

In a recent paper, Holloway and Ansari (in press) compared the developmental trajectory of the distance effect across several dimensions. They tested the comparison of numerical quantities presented as digits, and as visual arrays, as well as the comparison of height and luminance. As age increased (6-8 years old, and adults), the size of the distance effect decreased conjointly for all the types of comparisons tested, suggesting that this evolution reflects the development of a shared comparison process. However, these results do not preclude the possibility that different representations do exist, especially if one assumes that the distance effect does not reflect mental representation per se (see Section 2). Therefore, these results are in accord with the convergence observed in infants' discrimination of quantities across several dimensions.

In adults, the most conclusive evidence for a shared magnitude representation format arises from the interaction between numerical value and other magnitudes, such as physical length (e.g., the size congruity effect). These effects typically involve symbolic numerical representations, and thus cannot be studied in preverbal infants. In the years following their learning of Arabic digits, children start to develop the adult-like effects of interference between space and number. The SNARC effect has been observed in children aged 9 years old, but not in younger children (Berch et al., 1999). In the size congruity paradigm, children show a mutual interference between size and number starting at the end of first grade when they have to perform a magnitude comparison (Rubinsten et al., 2002). However, such a mutual interference is absent at the beginning of first grade (Girelli et al., 2000; Rubinsten et al., 2002). These results reflect the slow development of the automatic association between digits and the quantity they refer to (see Ansari et al., 2005, for the evolution of the neural basis of digits comparison in childhood). In order to assess whether number and other dimensions interfere in children from an early age, further research needs to investigate the presence of interference between number and other dimensions using nonsymbolic numerical stimuli (see Hurewitz et al., 2006, for a study with adults). A first step in this direction revealed that starting from the age of 5 years old, children process numerosity in an automatic manner which affects physical size comparison (Gebuis et al., submitted for publication).

\subsubsection{Evidence for shared representation from developmental synaesthesia}

A different way to examine characteristics of magnitude and numerical representations is to examine people with developmental synaesthesia. Synaesthesia is a case in which certain perceptual or conceptual stimuli (e.g., numbers) trigger an additional concurrent experience (e.g., color). Previous studies suggested that, due to the direct and conscious access to the 
mental representation, synaesthesia can serve as a valuable tool to inform cognitive theories (Cohen Kadosh and Henik, 2007). In line with this idea, it was found that people that experience numbers in colors (digit-color synaesthesia) have a tight correspondence between the number and the luminance in the corresponding color that is experienced (Cohen Kadosh et al., 2007f). Namely, the larger the numerical value, the lower the degree of the luminance. Importantly, this organization was based on cardinality (magnitude) rather than ordinality and follows the Weber-Fechner law, which has been reported previously for numerical representation in humans and monkeys (Dehaene, 2003). Notably, the correspondence between numerical and luminance values was not based on a comparison task. Rather, the tight link between these magnitudes was obtained by analyzing the digit and the corresponding color's components that the synaesthetes experienced in their everyday life. This correspondence is analogous to 2-year-old children who associate brightness with small objects and darkness with large objects (Smith and Sera, 1992). This might suggest a shared representation of magnitude in an early developmental stage, which is expressed later in life in the synaesthetic experience.

\section{Conclusions}

Behavioral studies suggest that numbers are probably not special, meaning that their magnitude is processed by means of domain-general magnitude representations. First, most of the behavioral effects found with numbers (e.g., distance effect, size effect, and SNARC effect) can also be obtained with nonnumerical magnitudes. Second, magnitudes based upon symbolic and non-symbolic quantities can interact and cause a mutual interference. All these findings suggest the existence of shared magnitude representation. Converging evidence for these behavioral results is coming from neuroimaging findings, which show shared activation patterns for different magnitudes in the IPS. On the other hand, part of the neuroimaging data also supports the existence of distinct magnitude mechanisms. In addition, other neuroimaging studies suggest the existence of both shared and distinct magnitude mechanisms, where task demands drive the usage of one or the other mechanism. The possible co-existence between both types of mechanisms, pointed out by studies involving human adults, raises the question of their developmental trajectories. However, few comparative and developmental studies have addressed this question. Although human infants and animals have been shown to process various magnitude dimensions in similar fashion, to date we do not possess very strong evidence to decide whether they evoke shared or specific representations, or both.

Future research in this field is critical for a basic understanding of the human and the primate brain, and to broaden our understanding of the neuronal substrates that might be impaired in some populations (e.g., developmental dyscalculia (Ansari and Karmiloff-Smith, 2002; Butterworth, 2004; Cohen Kadosh and Walsh, 2007; Cohen Kadosh et al., 2007d; Wilson and Dehaene, 2007)).

\section{Acknowledgements}

We wish to thank Kathrin Cohen Kadosh, Wim Fias, Desiree Meloul, and Vincent Walsh, the anonymous reviewers for their very helpful comments, and our colleagues of the European Diploma in Cognitive and Brain Sciences (EDCBS) group. This work was supported by grants from the International Brain Research Organization and Marie Curie Intra-European Fellowship (RCK), and the Fyssen Foundation (VI).

\section{References}

Algom, D., Dekel, A., Pansky, A., 1996. The perception of number from the separability of the stimulus: the Stroop effect revisited. Mem. Cogn. 24, 557-572.

Altman, D.G., Bland, J.M., 1995. Absence of evidence is not evidence of absence. BMJ 311, 485.

Ansari, D., Dhital, B., 2006. Age-related changes in the activation of the intraparietal sulcus during non-symbolic magnitude processing: an eventrelated functional magnetic resonance imaging study. J. Cogn. Neurosci. 18, 1820-1828.

Ansari, D., Dhital, B., Siong, S.C., 2006a. Parametric effects of numerical distance on the intraparietal sulcus during passive viewing of rapid numerosity changes. Brain Res. 1067, 181-188.

Ansari, D., Fugelsang, J.A., Dhital, B., Venkatraman, V., 2006b. Dissociating response conflict from numerical magnitude processing in the brain: an event-related fMRI study. Neuroimage 32, 799-805.

Ansari, D., Garcia, N., Lucas, E., Hamon, K., Dhital, B., 2005. Neural correlates of symbolic number processing in children and adults. Neuroreport 16, 1769-1773.

Ansari, D., Karmiloff-Smith, A., 2002. Atypical trajectories of number development: a neuroconstructivist perspective. Trends Cogn. Sci. 6, 511-516.

Bächtold, D., Baumüller, M., Brugger, P., 1998. Stimulus-response compatibility in representational space. Neuropsychologia 36, 731-735.

Barth, H., Le Mont, K., Lipton, J., Spelke, E.S., 2005. Abstract number and arithmetic in preschool children. Proc. Natl. Acad. Sci. U.S.A. 102, 1411614121.

Berch, D.B., Foley, E.J., Hill, R.J., Ryan, P.M., 1999. Extracting parity and magnitude from Arabic numerals: developmental changes in number processing and mental representation. J. Exp. Child Psychol. 74, 286-308.

Birren, J.E., Botwinick, J., 1955. Speed of response as a function of perceptual difficulty and age. J. Gerontol. 10, 433-436.

Bledowski, C., Prvulovic, D., Hoechstetter, K., Scherg, M., Wibral, M., Goebel, R., Linden, D.E.J., 2004. Localizing P300 generators in visual target and distractor processing: a combined event-related potentials and functional magnetic resonance imaging study. J. Neurosci. 24, 9353-9360.

Brannon, E.M., 2006. The representation of numerical magnitude. Curr. Opin. Neurobiol. 16, 222-229.

Brannon, E.M., Abbott, S., Lutz, D.J., 2004. Number bias for the discrimination of large visual sets in infancy. Cognition 93, B59-B68.

Brannon, E.M., Lutz, D., Cordes, S., 2006. The development of area discrimination and its implications for number representation in infancy. Dev. Sci. 9, F59-F64.

Brannon, E.M., Suanda, U., Libertus, K., 2007. Increasing precision in temporal discriminations over development parallels the development of number discrimination. Dev. Sci. 10, 770-777.

Brannon, E.M., Terrace, H.S., 1998. Ordering of the numerosities 1 to 9 by monkeys. Science 282, 746-749.

Buckley, P.B., Gillman, C.B., 1974. Comparisons of digits and dot patterns. J. Exp. Psychol. 103, 1131-1136.

Bunge, S.A., Hazeltine, E., Scanlon, M.D., Rosen, A.C., Gabrieli, J.D., 2002. Dissociable contributions of prefrontal and parietal cortices to response selection. Neuroimage 17, 1562-1571.

Butterworth, B., 1999. The Mathematical Brain. Macmillan, London, UK. 
Butterworth, B., 2004. Developmental dyscalculia. In: Campbell, J.I.D. (Ed.), Handbook of Mathematical Cognition. Psychology Press, New York, pp. 455-467.

Cantlon, J.F., Brannon, E.M., 2006. Shared system for ordering small and large numbers in monkeys and humans. Psychol. Sci. 17, 401-406.

Cantlon, J.F., Brannon, E.M., Carter, E.J., Pelphrey, K.A., 2006. Functional imaging of numerical processing in adults and 4-y-old children. PLoS Biol. 4, e125.

Carey, S., 2004. Bootstrapping and the origin of concepts. Daedalus Winter, 59 68.

Castelli, F., Glaser, D.E., Butterworth, B., 2006. Discrete and analogue quantity processing in the parietal lobe: a functional MRI study. Proc. Natl. Acad. Sci. U.S.A. 103, 4693-4698.

Chiao, J.Y., Bordeaux, A.R., Ambady, N., 2004. Mental representations of social status. Cognition 93, B49-B57.

Church, R.M., Meck, W.H., 1984. The numerical attribute of stimuli. In: Toitblat, H.L., Bever, T.G., Terrace, H.S. (Eds.), Animal Cognition. Erlbaum, Hillsdale, NJ.

Clearfield, M.W., Mix, K.S., 1999. Number versus contour length in infants' discrimination of small visual sets. Psychol. Sci. 10, 408-411.

Cohen Kadosh, K., Johnson, M.H., 2007. Developing a cortex specialized for face perception. Trends Cogn. Sci. 11, 367-369.

Cohen Kadosh, R., Brodsky, W., Levin, M., Henik, A., in press-a. Mental representation: what can pitch tell us about the distance effect? Cortex.

Cohen Kadosh, R., Cohen Kadosh, K., Henik, A., in press-b. When brightness counts: the neuronal correlate of numerical-luminance interference. Cereb. Cortex.

Cohen Kadosh, R., Tzelgov, J., Henik, A., in press-c. A synesthetic walk on the mental number line: the size effect. Cognition.

Cohen Kadosh, R., Cohen Kadosh, K., Henik, A., 2007a. The neuronal correlate of bi-directional synaesthesia: a combined ERP and fMRI study. J. Cogn. Neurosci. 19, 2050-2059.

Cohen Kadosh, R., Cohen Kadosh, K., Kaas, A., Henik, A., Goebel, R., 2007b. Notation-dependent and -independent representations of numbers in the parietal lobes. Neuron 53, 307-314.

Cohen Kadosh, R., Cohen Kadosh, K., Linden, D.E.J., Gevers, W., Berger, A., Henik, A., 2007c. The brain locus of interaction between number and size: a combined functional magnetic resonance imaging and event-related potential study. J. Cogn. Neurosci. 19, 957-970.

Cohen Kadosh, R., Cohen Kadosh, K., Schuhmann, T., Kaas, A., Goebel, R., Henik, A., Sack, A.T., 2007d. Virtual dyscalculia induced by parietallobe TMS impairs automatic magnitude processing. Curr. Biol. 17, 689-693.

Cohen Kadosh, R., Henik, A., Rubinsten, O., 2007e. The effect of orientation on number word processing. Acta Psychol. (Amst.) 124, 370-381.

Cohen Kadosh, R., Henik, A., Walsh, V., 2007f. Small is bright and big is dark in synaesthesia. Curr. Biol. 17, R834-R835.

Cohen Kadosh, R., Walsh, V., Henik, A., 2007g. Selecting between intelligent options. Behav. Brain Sci. 30, 155.

Cohen Kadosh, R., Henik, A., 2006. A common representation for semantic and physical properties: a cognitive-anatomical approach. Exp. Psychol. 53, 87-94.

Cohen Kadosh, R., Henik, A., 2007. Can synaesthesia research inform cognitive science? Trends Cogn. Sci. 11, 177-184.

Cohen Kadosh, R., Henik, A., Rubinsten, O., Mohr, H., Dori, H., Van de Ven, V., Zorzi, M., Hendler, T., Goebel, R., Linden, D.E.J., 2005. Are numbers special? The comparison systems of the human brain investigated by fMRI. Neuropsychologia 43, 1238-1248.

Cohen Kadosh, R., Walsh, V., 2007. Dyscalculia. Curr. Biol. 17, R946-R947.

Corbetta, M., Shulman, G.L., 2002. Control of goal-directed and stimulusdriven attention in the brain. Nat. Rev. Neurosci. 3, 201-215.

Crossman, E.R.F.W., 1955. The measurement of discriminability. Q. J. Exp. Psychol. 7, 176-195.

Dehaene, S., 1992. Varieties of numerical abilities. Cognition 44, 1-42.

Dehaene, S., 1997. The Number Sense: How the Mind Creates Mathematics. Oxford University Press, Oxford, UK.

Dehaene, S., 2003. The neural basis of the Weber-Fechner law: a logarithmic mental number line. Trends Cogn. Sci. 7, 145-147.
Dehaene, S., Bossini, S., Giraux, P., 1993. The mental representation of parity and number magnitude. J. Exp. Psychol. Gen. 122, 371-396.

Dehaene, S., Dehaene-Lambertz, G., Cohen, L., 1998. Abstract representations of numbers in the animal and human brain. Trends Neurosci. 21, $355-361$.

Dehaene, S., Molko, N., Cohen, L., Wilson, A.J., 2004. Arithmetic and the brain. Curr. Opin. Neurobiol. 14, 218-224.

Dehaene, S., Piazza, M., Pinel, P., Cohen, L., 2003. Three parietal circuits for number processing. Cogn. Neuropsychol. 20, 487-506.

Di Luca, S., Grana, A., Semenza, C., Seron, X., Pesenti, M., 2006. Finger-digit compatibility in Arabic numeral processing. Q. J. Exp. Psychol. 59, 16481663.

Dormal, V., Seron, X., Pesenti, M., 2006. Numerosity-duration interference: a Stroop experiment. Acta Psychol. (Amst.) 121, 109-124.

Eger, E., Sterzer, P., Russ, M.O., Giraud, A.-L., Kleinschmidt, A., 2003. A supramodal number representation in human intraparietal cortex. Neuron 37, 719-725.

Feigenson, L., 2005. A double-dissociation in infants' representations of object arrays. Cognition 95, B37-B48.

Feigenson, L., 2007. The equality of quantity. Trends Cogn. Sci. 11, 185-187.

Feigenson, L., Carey, S., Spelke, E., 2002. Infants' discrimination of number vs continuous extent. Cogn. Psychol. 44, 33-66.

Féron, J., Gentaz, E., Streri, A., 2006. Evidence of amodal representation of small numbers across visuo-tactile modalities in 5-month-old infants. Cogn. Dev. 21, 81-92.

Fias, W., 2001. Two routes for the processing of verbal numbers: evidence from the SNARC effect. Psychol. Res. 65, 250-259.

Fias, W., Brysbaert, M., Geypens, F., d'Ydewalle, G., 1996. The importance of magnitude information in numerical processing: evidence from the SNARC effect. Math. Cogn. 2, 95-110.

Fias, W., Dupont, P., Reynvoet, B., Orban, G.A., 2002. The quantitative nature of a visual task differentiates between ventral and dorsal stream. J. Cogn. Neurosci. 14, 646-658.

Fias, W., Fischer, M.H., 2004. Spatial representation of numbers. In: Campbell, J.I.D. (Ed.), Handbook of Mathematical Cognition. Psychology Press, New York, pp. 43-54.

Fias, W., Lammertyn, J., Reynvoet, B., Dupont, P., Orban, G.A., 2003. Parietal representation of symbolic and nonsymbolic magnitude. J. Cogn. Neurosci. $15,1-11$.

Fias, W., Lauwereyns, J., Lammertyn, J., 2001. Irrelevant digits affect featurebased attention depending on the overlap of neural circuits. Brain Res. Cogn. Brain Res. 12, 415-423.

Fischer, M.H., Castel, A.D., Dodd, M.D., Pratt, J., 2003. Perceiving numbers causes spatial shifts of attention. Nat. Neurosci. 6, 555-556.

Fischer, M.H., Rottmann, J., 2005. Do negative numbers have a place on the mental number line? Psychol. Sci. 47, 22-32.

Fulbright, R.K., Manson, S.C., Skudlarski, P., Lacadie, C.M., Gore, J.C., 2003. Quantity determination and the distance effect with letters, numbers, and shapes: a functional MR imaging study of number processing. Am. J. Neuroradiol. 23, 193-200.

Gallistel, C.R., Gelman, R., 1992. Preverbal and verbal counting and computation. Cognition 44, 43-74.

Gallistel, C.R., Gelman, R., 2004. Mathematical cognition. In: Campbell, J.I.D. (Ed.), Handbook of Mathematical Cognition. Psychology Press, New York.

Geary, D.G., 1993. Mathematical disabilities: cognitive, neuropsychological, and genetic components. Psychol. Bull. 114, 345-362.

Gebuis, T., Cohen Kadosh, R., de Haan, E., Henik, A., submitted for publication. Automatic quantity processing in 5 -year olds.

Gelman, R., Butterworth, B., 2005. Number and language: how are they related? Trends Cogn. Sci. 9, 6-10.

Gevers, W., Lammertyn, J., 2005. The hunt for SNARC. Psychol. Sci. 47, $10-21$.

Gevers, W., Reynvoet, B., Fias, W., 2003. The mental representation of ordinal sequences is spatially organized. Cognition 87, B87-B95.

Gevers, W., Reynvoet, B., Fias, W., 2004. The mental representation of ordinal sequences is spatially organised: evidence from days of the week. Cortex 40 , $171-172$. 
Girelli, L., Lucangeli, D., Butterworth, B., 2000. The development of automaticity in accessing number magnitude. J. Exp. Child Psychol. $76,104-122$.

Göbel, S.M., Johansen-Berg, H., Behrens, T., Rushworth, M.F.S., 2004. Response-selection-related parietal activation during number comparison. J. Cogn. Neurosci. 16 (9), 1536-1551.

Gordon, P., 2004. Numerical cognition without words: evidence from Amazonia. Science 306, 496-499.

Gratton, G., Coles, M.G.H., Sirevaag, E.J., Eriksen, C.W., Donchin, E., 1988. Pre- and poststimulus activation of response channels: a psychophysiological analysis. J. Exp. Psychol. Hum. Percept. Perform. 14, 331-344.

Grill-Spector, K., Henson, R., Martin, A., 2006. Repetition and the brain: neural models of stimulus-specific effects. Trends Cogn. Sci. 10, 14-23.

Grill-Spector, K., Malach, R., 2001. FMR-adaptation: a tool for studying the functional properties of human cortical neurons. Acta Psychol. (Amst.) 107, 293-321.

Henik, A., Tzelgov, J., 1982. Is three greater than five: the relation between physical and semantic size in comparison tasks. Mem. Cogn. 10, 389-395.

Henmon, V.A.C., 1906. The time of perception as a measure of differences in sensation. Arch. Philos. Psychol. Sci. Meth. 8, 5-75.

Holloway, I., Ansari, D., in press. Domain-specific and domain-general changes in children's development of number comparison. Dev. Sci.

Hubbard, E.M., Piazza, M., Pinel, P., Dehaene, S., 2005. Interactions between number and space in parietal cortex. Nat. Rev. Neurosci. 6, 435-448.

Huntley-Fenner, G., Carey, S., Solimando, A., 2002. Objects are individuals but stuff doesn't count: perceived rigidity and cohesiveness influence infants' representations of small groups of discrete entities. Cognition 85, 203-221.

Hurewitz, F., Gelman, R., Schnitzer, B., 2006. Sometimes area counts more than number. Proc. Natl. Acad. Sci. U.S.A. 103, 19599-19604.

Jiang, Y., Kanwisher, N., 2003. Common neural substrates for response selection across modalities and mapping paradigms. J. Cogn. Neurosci. 15, 1080-1094.

Johnson, D.M., 1939. Confidence and speed in the two-category judgment. Arch. Psychol. 241, 1-52.

Johnson, M.H., 2001. Functional brain development in humans. Nat. Rev. Neurosci. 2, 475-483.

Kansaku, K., Johnson, A., Grillon, M.-L., Garraux, G., Sadato, N., Hallett, M., 2006. Neural correlates of counting of sequential sensory and motor events in the human brain. Neuroimage 31, 649-660.

Kaufmann, L., Koppelstaetter, F., Delazer, M., Siedentopf, C., Rhomberg, P., Golaszewski, S., Felber, S., Ischebeck, A., 2005. Neural correlates of distance and congruity effects in a numerical Stroop task: an event-related fMRI study. Neuroimage 25, 888-898.

Kobayashi, T., Hiraki, K., Hasegawa, T., 2005. Auditory-visual intermodal matching of small numerosities in 6-month-old infants. Dev. Sci. 8, 409419.

Kok, A., 2001. On the utility of P3 amplitude as a measure of processing capacity. Psychophysiology 38, 557-577.

Lammertyn, J., Fias, W., Lauwereyns, J., 2002. Semantic influences on featurebased attention due to overlap of neural circuits. Cortex 38, 878-882.

Leon, M.I., Gallistel, C.R., 1998. Self-stimulating rats combine subjective reward magnitude and subjective reward rate multiplicatively. J. Exp. Psychol. Anim. Behav. Process. 24, 265-277.

Linden, D.E.J., 2005. The P300: where in the brain is it produced and what does it tell us? Neuroscientist 11, 563-576.

Lipton, J.S., Spelke, E.S., 2003. Origins of number sense: large-number discrimination in human infants. Psychol. Sci. 14, 396-401.

McCloskey, M., 1992. Cognitive mechanisms in numerical processing: evidence from acquired dyscalculia. Cognition 44, 107-157.

McCrink, K., Wynn, K., 2004. Large-number addition and subtraction in infants. Psychol. Sci. 15, 776-781.

Meck, W.H., Church, R.M., 1983. A mode control model of counting and timing process. J. Exp. Psychol. Anim. Behav. Process. 9, 320-334.

Mix, K.S., Huttenlocher, J., Levine, S.C., 2002. Multiple cues for quantification in infancy: is number one of them? Psychol. Bull. 128, 278-294.

Mix, K.S., Levine, S.C., Huttenlocher, J., 1997. Numerical abstraction by infants: another look. Dev. Psychol. 32, 423-428.
Molko, N., Cachia, A., Rivière, D., Mangin, J.F., Bruandet, M., Le Bihan, D., Cohen, L., Dehaene, S., 2003. Functional and structural alterations of the intraparietal sulcus in a developmental dyscalculia of genetic origin. Neuron $40,847-858$.

Moore, D., Benenson, J., Reznick, J.S., Peterson, M., Kagan, J., 1987. Effect of auditory numerical information on infants' looking behavior: contradictory evidence. Dev. Psychol. 23, 665-670.

Moyer, R.S., Landauer, T.K., 1967. Time required for judgment of numerical inequality. Nature 215, 1519-1520.

Nieder, A., 2004. The number domain — can we count on parietal cortex? Neuron 44, 407-409.

Nieder, A., 2005. Counting on neurons: the neurobiology of numerical competence. Nat. Rev. Neurosci. 6, 177-190.

Nieder, A., Diester, I., Tuduscius, O., 2006. Temporal and spatial enumeration processes in the primate parietal cortex. Science 313, 1431-1435.

Nieder, A., Freedman, D.J., Miller, E.K., 2002. Representation of the quantity of visual items in the primate prefrontal cortex. Science 297, 1708-1711.

Nieder, A., Miller, E.K., 2003. Coding of cognitive magnitude: compressed scaling of numerical information in the primate prefrontal cortex. Neuron 37, 149-157.

Nieder, A., Miller, E.K., 2004. A parieto-frontal network for visual numerical information in the monkey. Proc. Natl. Acad. Sci. U.S.A. 101, 7457-7462.

Orban, G.A., Dupont, P., Vogels, R., De Bruyn, B., Bormans, G., Mortelmans, L., 1996. Task dependency of visual processing in the human visual system. Behav. Brain Res. 76, 215-223.

Pansky, A., Algom, D., 1999. Stroop and Garner effects in comparative judgment of numerals: the role of attention. J. Exp. Psychol. Hum. Percept. Perform. 25, 39-58.

Parkman, J.M., 1971. Temporal aspects of digit and letter inequality judgments. J. Exp. Psychol. 91, 191-205.

Pascual-Leone, A., Amedi, A., Fregni, F., Merabet, L.B., 2005. The plastic human brain cortex. Annu. Rev. Neurosci. 28, 377-401.

Piaget, J., 1952. The Child's Conception of Number. Norton, New York.

Piazza, M., Izard, V., Pinel, P., Le Bihan, D., Dehaene, S., 2004. Tuning curves for approximate numerosity in the human intraparietal sulcus. Neuron 44 , 547-555.

Piazza, M., Pinel, P., Le Bihan, D., Dehaene, S., 2007. A magnitude code common to numerosities and number symbols in human intraparietal cortex. Neuron 53, 293-305.

Pica, P., Lemer, C., Izard, V., Dehaene, S., 2004. Exact and approximate arithmetic in Amazonian indigene group. Science 306, 499-503.

Pinel, P., Piazza, M., Le Bihan, D., Dehaene, S., 2004. Distributed and overlapping cerebral representations of number, size, and luminance during comparative judgments. Neuron 41, 983-993.

Pinhas, M., Tzelgov, J., Guata, I., submitted for publication. The size effect reflects a basic feature of the mental number line.

Poldrack, R.A., 2000. Imaging brain plasticity: conceptual and methodological issues-a theoretical review. Neuroimage 12, 1-13.

Restle, F., 1970. Speed of adding and comparing numbers. J. Exp. Psychol. 83, 274-278.

Rivera, S.M., Reiss, A.L., Eckert, M.A., Menon, V., 2005. Developmental changes in mental arithmetic: evidence for increased functional specialization of the left inferior parietal cortex. Cereb. Cortex 15, 1779-1790.

Roitman, J.D., Brannon, E.M., Andrews, J.R., Platt, M.L., 2007. Nonverbal representation of time and number in adults. Acta Psychol. (Amst.) 124, 296-318.

Rourke, B.P., 1993. Arithmetic disabilities, specific and otherwise: a neuropsychological perspective. J. Learn. Disabil. 26, 214-226.

Rubinsten, O., Henik, A., Berger, A., Shahar-Shalev, S., 2002. The development of internal representations of magnitude and their association with Arabic numerals. J. Exp. Child Psychol. 81, 74-92.

Rumelhart, D.E., McClelland, J.L., 1986. Parallel Distributed Processing: Explorations in the Microstructure of Cognition. MIT Press, Cambridge, MA.

Rusconi, E., Kwan, B., Giordano, B.L., Umilta, C., Butterworth, B., 2006. Spatial representation of pitch height: the SMARC effect. Cognition 99, $113-129$. 
Schwarz, W., Heinze, H.J., 1998. On the interaction of numerical and size information in digit comparison: a behavioral and event-related potential study. Neuropsychologia 36, 1167-1179.

Schwarz, W., Ischebeck, A., 2003. On the relative speed account of the numbersize interference in comparative judgment of numerals. J. Exp. Psychol Hum. Percept. Perform. 29, 507-522.

Shaki, S., Petrusic, W.M., 2005. On the mental representation of negative numbers: context-dependent SNARC effects with comparative judgments. Psychon. Bull. Rev. 12, 931-937.

Shuman, M., Kanwisher, N., 2004. Numerical magnitude in the human parietal lobe: tests of representational generality and domain specificity. Neuron 44, $557-569$.

Simon, O., Mangin, J.F., Cohen, L., Le Bihan, D., Dehaene, S., 2002. Topographical layout of hand, eye, calculation, and language-related areas in the human parietal lobe. Neuron 33, 475-487.

Smith, L.B., Sera, M.D., 1992. A developmental analysis of the polar structure of dimensions. Cogn. Psychol. 24, 142-199.

Starkey, P., Spelke, E.S., Gelman, R., 1983. Detection of intermodal numerical correspondences by human infants. Science 222, 179-181.

Starkey, P., Spelke, E.S., Gelman, R., 1990. Numerical abstraction by human infants. Cognition 36, 97-128.

Talairach, J., Tournoux, P., 1988. Co-planar Stereotaxic Atlas of the Human Brain. Thieme, New York.

Tang, J., Critchley, H.D., Glaser, D., Dolan, R.J., Butterworth, B., 2006 Imaging informational conflict: a functional magnetic resonance imaging study of numerical Stroop. J. Cogn. Neurosci. 18, 2049-2062.

Tuduscius, O., Nieder, A., 2007. Neuronal population coding of continuous and discrete quantity in the primate posterior parietal cortex. Proc. Natl. Acad. Sci. U.S.A. 104, 14513-14518.

Tzelgov, J., Meyer, J., Henik, A., 1992. Automatic and intentional processing of numerical information. J. Exp. Psychol.: Learn. Mem. Cogn. 18, 166-179.

Urton, G., Brezine, C., 2005. Khipu accounting in ancient Peru. Science 309, 1065-1067.

Van Essen, D.C., 2002. Windows on the brain. The emerging role of atlases and databases in neuroscience. Curr. Opin. Neurobiol. 12, 574-579.
Van Essen, D.C., Dickson, J., Harwell, J., Hanlon, D., Anderson, C.H., Drury, H.A., 2001. An integrated software system for surface-based analyses of cerebral cortex. J. Am. Med. Inform. Assoc. 8, 443-459.

vanMarle, K., Wynn, K., 2006. Six-month-old infants use analog magnitudes to represent duration. Dev. Sci. 9, F41-F49.

Verguts, T., Fias, W., Stevens, M., 2005. A model of exact small-number representation. Psychon. Bull. Rev. 12, 66-80.

Vuilleumier, P., Ortigue, S., Brugger, P., 2004. The number space and neglect. Cortex 40, 399-410.

Walsh, V., 2003. A theory of magnitude: common cortical metrics of time, space and quantity. Trends Cogn. Sci. 7, 483-488.

Welford, A.T., 1960. The measurement of sensory-motor performance: survey and reappraisal of twelve years' progress. Ergonomics 3, 189-230.

Wiese, H., 2003. Iconic and non-iconic stages in number development: the role of language. Trends Cogn. Sci. 7, 385-390.

Wilkinson, D., Halligan, P., 2004. The relevance of behavioural measures for functional-imaging studies of cognition. Nat. Rev. Neurosci. 5, 67-73.

Wilson, A.J., Dehaene, S., 2007. Number sense and developmental dyscalculia. In: Coch, D., Dawson, G., Fischer, K. (Eds.), Human Behavior, Learning, and the Developing Brain: Atypical Development. Guilford Press, New York.

Wynn, K., 1992. Addition and subtraction by human infants. Nature 358, 749750.

Xu, F., Arriaga, R.I., 2007. Number discrimination in 10-month-old infants. Br. J. Dev. Psychol. 25, 103-108.

$\mathrm{Xu}$, F., Spelke, E.S., 2000. Large number discrimination in 6-month-old infants. Cognition 74, B1-B11.

Zebian, S., 2005. Linkages between number concepts, spatial thinking, and directionality of writing: the SNARC effect and the reverse SNARC effect in English and Arabic monoliterates, biliterates and illiterate Arabic speakers. J. Cogn. Cult. 5, 165-191.

Zorzi, M., Priftis, K., Umilta, C., 2002. Neglect disrupts the mental number line. Nature 417, 138-139.

Zorzi, M., Butterworth, B., 1999. A Computational Model of Number Comparison. Erlbaum, Mahwah (NJ). 